\title{
Physicochemical properties, in vitro release and in vivo evaluation of tramadol hydrochloride rectal suppository and rectal gel
}

\author{
Wantana Reanmongkol ${ }^{\mathrm{a}}$, Nattha Kaewnopparat ${ }^{\mathrm{b}}$, Chaveewan Ratanajamit ${ }^{\mathrm{a}}$ \\ ${ }^{a}$ Department of Clinical Pharmacy; ${ }^{b}$ Department of Pharmaceutical Technology, Faculty of \\ Pharmaceutical Sciences, Prince of Songkla University, Hat Yai, Songkhla 90110, Thailand
}

\begin{abstract}
Background: Tramadol is a centrally acting analgesic drug. Rectal administration of tramadol is useful in the treatment of post-operative pain or malignant pain in cases where it cannot be administered orally. In Thailand, tramadol is available only as a capsule for oral use and as a solution for injection.

Objective: Develop tramadol hydrochloride rectal suppositories and rectal gel preparations.

Methods: Tramadol rectal suppository and rectal gel were prepared. Physicochemical properties (viscosity, gel strength, mucoadhesive force) and the in vitro release of tramadol hydrochloride were investigated from different bases (Witepsol H15, polyethylene glycol, poloxamer, and hydroxyethylcellulose). The analgesic activity of rectal tramadol hydrochloride using the hot plate test was evaluated in rats.

Results: Tramadol hydrochloride rectal gel using poloxamer was more mucoadhesive to the rectal mucous membrane than was the gel with the hydroxyethylcellulose base. Tramadol hydrochloride was released rapidly in vitro from both the Witepsol H15 and polyethylene glycol bases. It was completely released from the polyethylene glycol suppository base within 15 minutes. The amount of tramadol hydrochloride release from the Witepsol H15 suppository base was about $93 \%$ at 120 minutes. When using poloxamer or hydroxyethylcellulose as a rectal base, tramadol hydrochloride was released from both bases rapidly and completely released within 15 minutes. Administration of a tramadol hydrochloride suppository in rats exhibited a more pronounced analgesic effect with the polyethylene glycol base than with the Witepsol H15-based suppositories. The rectal gel had a less pronounced analgesic effect when made with the hydroxyethylcellulose base than with the poloxamer base.

Conclusion: Tramadol hydrochloride suppositories and rectal gels with different bases showed rapid and almost complete drug release from the bases, prolonging the latency of a nociceptive response in in vivo experiments.
\end{abstract}

Keywords: Analgesic, rectal gel, release study, suppository, tramadol

Tramadol is a synthetic 4-phenyl-piperidine analogue of codeine. It is a centrally acting analgesic with a low affinity for mu opioid receptors (a class of opioid receptors with high affinity for enkephalins and beta-endorphin but low affinity for dynorphins). It consists of two enantiomers, both of which contribute to its analgesic activity via different mechanisms. (+)Tramadol and its metabolite $(+)-O$-desmethyl-

Correspondence to: Wantana Reanmongkol, $\mathrm{PhD}$, Department of Clinical Pharmacy, Faculty of Pharmaceutical Sciences, Prince of Songkla University, Hat Yai, Songkhla 90110, Thailand. E-mail:wantana.r@psu.ac.th tramodol (M1), produced by liver $O$-demethylation, are agonists of the mu opioid receptor. $(+)$-Tramadol also inhibits serotonin reuptake whereas (-)-tramadol inhibits norepinephrine reuptake, to enhance the inhibition of pain $[1,2]$. Its use is indicated for the management of moderate to moderately severe pain including chronic pain and pain associated with molar extraction in adults [3].

Tramadol is an effective and well-tolerated agent to reduce pain resulting from trauma, renal or biliary colic, and labor, and for the management of chronic pain of malignant or nonmalignant origin, particularly neuropathic pain. Tramadol appears to produce less 
constipation and dependence than equianalgesic doses of strong opioids [2]. However, after intravenous and oral administration, peak concentrations are reached rapidly, and this has been associated with postoperative nausea and vomiting [4]. Thus, this limits the use of tramadol as a postoperative analgesic, especially in day surgery. Rectal administration of tramadol may be an alternative in this situation. It could be used at the same dose range as that of the oral form to maintain effective pain relief [5]. Furthermore, it is useful in the treatment of post-operative pain or malignant pain in cases where oral administration is not possible [6]. In Thailand, tramadol is not available in a form recommended for rectal administration. It is available only as a capsule for oral use and as a solution for injection [7].

In this study, we developed tramadol hydrochloride rectal suppository and rectal gel preparations. A rectal tramadol hydrochloride preparation was developed in two dosage forms, a rectal suppository and a rectal gel. Physicochemical properties (viscosity, gel strength, mucoadhesive force) and the in vitro release of tramadol hydrochloride from different bases were investigated on different bases (Witepsol H15, polyethylene glycol, poloxamer, and hydroxyethylcellulose). In addition, the analgesic activity of rectal tramadol hydrochloride was evaluated in rats.

\section{Materials and methods}

Tramadol hydrochloride was provided by Biolab Co, Ltd (Bangkok, Thailand). Poloxamer 188, poloxamer 407 , polyethylene glycol 400 , and polyethylene glycol 4000 were donated by BASF Aktiengesellschaft (Lugwidshafen, Germany), Witepsol H15 (Sciencelab, Houston, USA), hydroxyethylcellulose 4000 (Srichand United Dispensary Co, Bangkok, Thailand) were used. All chemicals were of reagent grade.

\section{Preparation of tramadol hydrochloride rectal suppository and gel}

Two formulations of tramadol rectal suppository were prepared by a fusion method using polyethylene glycol, Witepsol H 15, as suppository base. The amount of drug was $2 \mathrm{mg} / 2 \mathrm{~g}$ mold. Briefly, polyethylene glycol was heated to $55^{\circ} \mathrm{C}$. Then, pulverized tramadol hydrochloride was slowly added to the melted base with continuous agitation until a homogeneous mass was produced. Then, the resulting mixture was poured into a suppository mold at a temperature just above the congealing point of the suppository base and allowed to cool to $25^{\circ} \mathrm{C}$ until the mixture was solidified. The obtained suppositories were stored at $8-10^{\circ} \mathrm{C}$ until use.

Two formulations of tramadol hydrochloride rectal gel were prepared using poloxamer or hydroxyethylcellulose as the gel base. The amount of drug was $2 \mathrm{mg} / 0.2 \mathrm{~mL}$ of rectal gel. The poloxamer gel base consists of $15 \%$ poloxamer $407,12 \%$ poloxamer $188,5 \%$ propylene glycol, and purified water to $100 \%$. The tramadol hydrochloride rectal gel using poloxamer as a gel base was prepared by dissolving tramadol hydrochloride, propylene glycol and poloxamer in cold water and leaving overnight in the refrigerator. The hydroxyethylcellulose gel base consists of $0.75 \%$ hydroxyethylcellulose $4000,5 \%$ propylene glycol, and purified water to $100 \%$. The tramadol hydrochloride rectal gel using hydroxyethylcellulose as a gel base was prepared by dispersing hydroxyethylcellulose in water and heating in a water bath at $45^{\circ} \mathrm{C}$ with continuous stirring until a clear gel was obtained. Then, tramadol hydrochloride and propylene glycol were added to the gel base with continuous stirring.

\section{Measurement of physicochemical properties}

\section{pH and viscosity}

The $\mathrm{pH}$ and viscosity of prepared rectal gels were determined with a $\mathrm{pH}$ meter and Brookfield viscometer (Middleboro, USA), respectively.

\section{Gel strength}

This property was measured by the method described by Yong et al. [9] with a slight modification. Each tramadol hydrochloride rectal gel $(50 \mathrm{~g})$ was added into a $100 \mathrm{~mL}$ cylinder, and equilibrated in a water bath at $37.0^{\circ} \mathrm{C} \pm 0.5^{\circ} \mathrm{C}$ for 30 minutes. After placing a gel-strength device $(1.5 \mathrm{~cm}$ diameter, weight $25 \mathrm{~g}$ ) on the surface of gel base, the time (second) that the device took to move down through the cylinder for a distance of $5 \mathrm{~cm}$ was measured.

\section{Bioadhesive force}

The bioadhesive force of tramadol hydrochloride rectal gels was determined by using a mucoadhesive force device according to the method described by Yong et al. [9]. In brief, a section of tissue was cut from the fundus of a porcine rectum and instantly 
secured with the mucosal side facing out from a glass vial using a rubber band and an aluminum cap. The diameter of each exposed mucosal membrane was $2.5 \mathrm{~cm}$. One vial with a section of tissue attached was connected to a balance and another vial was placed on a height-adjustable pan. Tramadol rectal gel was spread onto the rectal tissue on the other vial. Then, the height of the vial was adjusted so that the tramadol rectal gel could be placed between the mucosal tissues of both vials. The weights were kept raised until the two vials got detached. The bioadhesive force, the detachment stress $\left(\right.$ dyne $\left./ \mathrm{cm}^{2}\right)$, was determined from the minimal weights that detached the two vials. The minimal weight that detached the two vials was recorded and calculated by using the equation, $\mathrm{F}=980 \mathrm{~m} / \pi \mathrm{r}^{2}$, where $\mathrm{m}$ and $\mathrm{r}$ represent the weight $(\mathrm{g})$ and radius of the mucosal membrane $(\mathrm{cm})$. The rectal tissue pieces were changed for each measurement.

\section{In vitro release experiment}

The in vitro release of tramadol hydrochloride from rectal formulations was determined. Purified water $(100 \mathrm{~mL})$ was used as the dissolution medium. A $2 \mathrm{~mL}$ sample was withdrawn, filtered and the amount of tramadol hydrochloride was determined by ultraviolet spectrophotometry (Spectronic Genesys 5, Milton Roy, Rochester, USA) at $271 \mathrm{~nm}$ using appropriate blank solutions. The tramadol hydrochloride concentration was calculated and expressed as a percentage of the drug released from the mean of six determinations.

\section{In vivo analgesic activity}

Male Wistar rats weighing $150 \pm 10 \mathrm{~g}$ were used for this study. The animals were housed for at least one week and fasted for 12 hours in the laboratory animal room prior to testing. All experimental protocols were approved by the Animal Ethics Committee, Prince of Songkla University.

The hot plate test was carried out according to the method described by Woolfe and MacDonald [10]. Rats were placed on a hot plate (Harvard Apparatus, Kent, UK) maintained at $55^{\circ} \mathrm{C} \pm 1{ }^{\circ} \mathrm{C}$. Latency of a nociceptive response such as licking of a hind limb or jumping was measured. Starting 30 minutes after rectal administration of the tramadol $(2 \mathrm{mg} /$ minisuppository or $2 \mathrm{mg} / 0.2 \mathrm{~mL}$ rectal gel), the nociceptive response was measured at 30 minutes and then every one hour over a four hour period. The cut-off time was 45 seconds. Only rats showing nociceptive responses within 15 seconds were used for the experiments.

\section{Statistical analysis}

Data were expressed as a mean standard error of mean (SEM), and were analyzed statistically by one-way ANOVA procedures, followed by Dunnett's test. A significant difference was considered at $\mathrm{p}<0.05$.

\section{Results}

\section{Physical properties}

The disintegration time for the tramadol hydrochloride suppository using Witepsol H15 or polyethylene glycol as the base was $9.0 \pm 0.4$ minutes and $21.0 \pm 0.5$ minutes, respectively. For the rectal gel, poloxamer and hydroxyethylcellulose were used as rectal gel bases. All prepared rectal gels were clear, colorless, viscous liquid with a $\mathrm{pH}$ range of 6.65-7.13. Figure 1 shows the viscosity of tramadol hydrochloride rectal gel using poloxamer and hydroxyethylcellulose base at room temperature and at $37^{\circ} \mathrm{C}$. Tramadol hydrochloride rectal gel using poloxamer at room temperature showed very low viscosity and a Newtonian flow, while at $37^{\circ} \mathrm{C}$, the viscosity increased markedly. Tramadol hydrochloride rectal gel using hydroxyethylcellulose base exhibited a high viscosity with non-Newtonian behavior.

\section{Gel strength}

The gel strength of the tramadol hydrochloride at the dose of $2 \mathrm{mg} / 0.2 \mathrm{~mL}$ using either poloxamer or hydroxyethylcellulose was 95 and 72 seconds, respectively. The tramadol hydrochloride rectal gel using poloxamer as the gel base has a higher gel strength than that when using hydroxyethylcellulose as the gel base.

\section{Bioadhesive force}

The mucoadhesive force of the tramadol hydrochloride rectal gel was $70.5 \times 10^{2}$ and $41.1 \times 10^{2}$ dyne $/ \mathrm{cm}^{2}$ for the poloxamer or hydroxyethylcellulose, respectively. Interestingly, the poloxamer base was more mucoadhesive to the rectal mucous membrane than that of hydroxyethylcellulose base. 


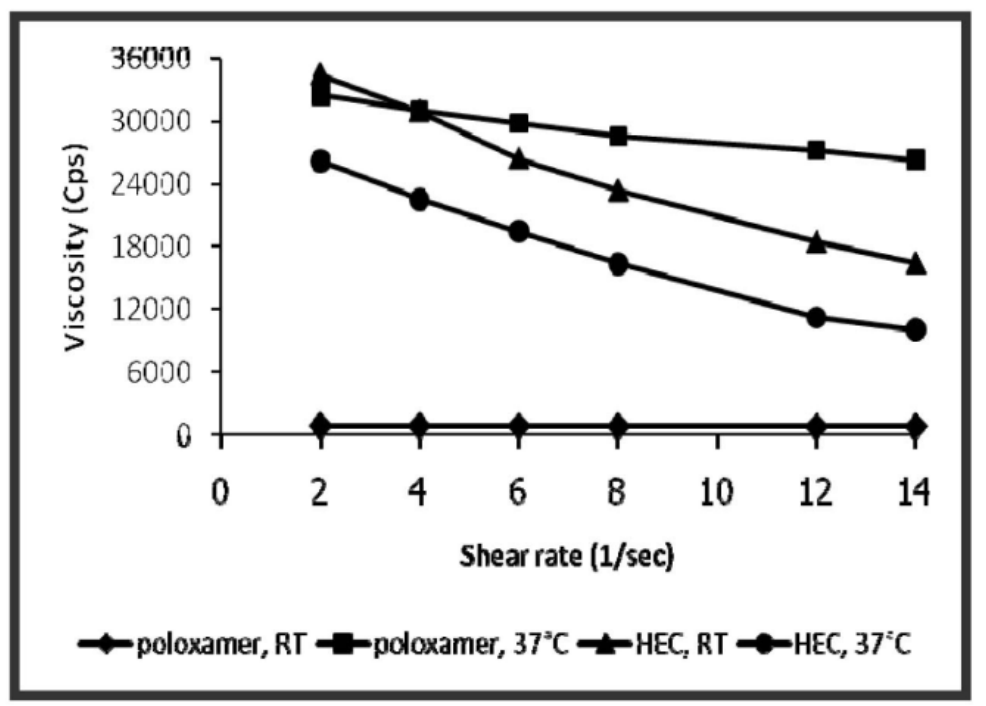

Figure 1. Viscosity of tramadol hydrochloride rectal gel using poloxamer and hydroxyethylcellulose as the gel base at room temperature $(\mathrm{RT})$ and at $37^{\circ} \mathrm{C}$.

\section{In vitro release study}

Figure 2 shows the percentage of tramadol hydrochloride released from the suppository bases at various time intervals at $37^{\circ} \mathrm{C}$. Tramadol hydrochloride was released rapidly from both of the bases within 15-30 minutes. Tramadol hydrochloride was completely released from polyethylene glycol suppository base within 15 minutes. Tramadol hydrochloride was also released rapidly from the Witepsol H15 suppository base but the amount of tramadol hydrochloride release from the suppository base was about $93 \%$ at 120 minutes.

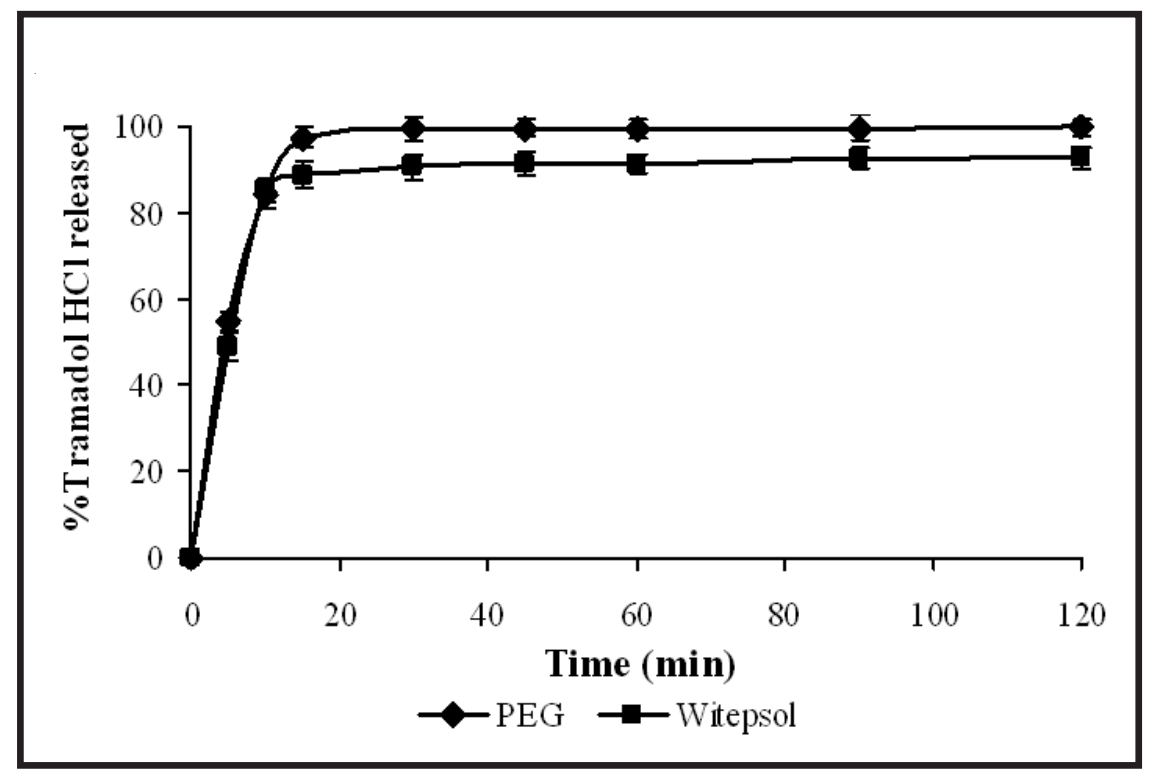

Figure 2. Percentage of tramadol hydrochloride released from polyethylene glycol (PEG) or Witepsol H15 suppository bases at various times. Each value represents the mean of six determinations. 
Using poloxamer and hydroxyethylcellulose as rectal gel bases, tramadol hydrochloride was released from both bases rapidly and completely released within 15 minutes.

\section{Analgesic activity in rats}

Table 1 shows latency of nociceptive response after administration of rectal tramadol hydrochloride using Witepsol H15, PEG, poloxamer, and HEC bases in rats. Rectal administration of tramadol hydrochloride ( $2 \mathrm{mg}$ ) in the suppository form using Witepsol H15 or polyethylene glycol suppository base significantly prolonged the latency of the nociceptive response in rats, but there was slightly more pronounced analgesic effect with the polyethylene glycol base than with the Witepsol H15 based suppositories in the rats. For the rectal gel, the results showed that administration of tramadol hydrochloride at the same dose $(2 \mathrm{mg})$ using poloxamer as gel base, exerted a marked increase of the latency of the nociceptive response in rats. Similar results were also observed when using hydroxyethylcellulose as base. It also significantly increased the nociceptive threshold after being subjected to heat, but there was slightly less pronounced analgesic effect with the hydroxyethylcellulose base than with the poloxamer based rectal gel in the rats.

\section{Discussion}

In the present study, two different suppository bases (Witepsol H15 and polyethylene glycol suppository bases) were used for rectal tramadol hydrochloride preparations. For disintegration according to the BP requirement [8], disintegration occurs in not more than 30 minutes for a lipophilicbased suppository and in not more than 60 minutes for a water-soluble suppository. Hence, all prepared suppositories were found to satisfy the BP requirement for disintegration.

For rectal gel, poloxamer base exhibited a gelation temperature at $34-35^{\circ} \mathrm{C}$, showing an enhanced bioavailability of the drugs [11]. This formulation exists as a liquid in vitro but a gel in vivo, and has a mucoadhesive property. We showed that this formulation was a little viscous with a Newtonian property at room temperature, while at $37^{\circ} \mathrm{C}$, its viscosity was markedly increased. This is due to the property of the poloxamer that changes from liquid into gel at body temperature $[12,13]$. In fact, with temperature increasing, the poloxamer becomes more viscous with configuration transformation from zigzag to a close-packed meander [14]. Therefore, after administering this preparation to the rectal cavity, it may be easily dispersed in the rectum, turning into a gel with high viscosity at physiological temperature. It probably attached well to the rectal mucous membrane without leakage. Rectal gel using hydroxyethylcellulose as the gel base showed a high viscosity and a nonNewtonian behavior.

Gel strength is an important parameter for finding the suitable condition without leakage from the anus after insertion [15]. The tramadol hydrochloride rectal gel using poloxamer as the gel base has a higher gel strength compared with using hydroxyethylcellulose as the gel base.

The mucoadhesive rectal gel can attach well in the rectal mucous membrane [16]. In the present

Table 1. Latency of nociceptive response versus time after administration of rectal tramadol hydrochloride using different bases in rats.

\begin{tabular}{|c|c|c|c|c|c|c|c|}
\hline \multirow[t]{2}{*}{ Base } & \multirow{2}{*}{$\begin{array}{l}\text { Tramadol } \\
\text { (mg) }\end{array}$} & \multicolumn{6}{|c|}{ Latency of nociceptive response (sec) } \\
\hline & & 0 hour & 0.5 hour & 1 hour & 2 hours & 3 hours & 4 hours \\
\hline \multirow[t]{2}{*}{ Witepsol H15 } & 0 & $3.45 \pm 0.50$ & $4.50 \pm 0.21$ & $4.58 \pm 0.30$ & $4.98 \pm 0.32$ & $4.40 \pm 0.47$ & $4.60 \pm 0.29$ \\
\hline & 2 & $3.65 \pm 0.27$ & $5.33 \pm 0.60$ & $5.80 \pm 0.28 *$ & $6.20 \pm 0.43 *$ & $6.15 \pm 0.50 *$ & $7.38 \pm 0.34 *$ \\
\hline \multirow[t]{2}{*}{ PEG } & 0 & $4.33 \pm 0.09$ & $4.40 \pm 0.14$ & $4.28 \pm 0.46$ & $4.83 \pm 0.15$ & $4.95 \pm 0.30$ & $4.55 \pm 0.17$ \\
\hline & 2 & $3.83 \pm 0.50$ & $6.63 \pm 0.13 *$ & $7.00 \pm 0.61 *$ & $7.83 \pm 0.35 *$ & $7.65 \pm 0.71 *$ & $7.43 \pm 0.63 *$ \\
\hline \multirow[t]{2}{*}{ Poloxamer } & 0 & $3.65 \pm 0.17$ & $4.18 \pm 0.22$ & $4.13 \pm 0.20$ & $3.80 \pm 0.32$ & $4.48 \pm 0.33$ & $4.18 \pm 0.27$ \\
\hline & 2 & $2.98 \pm 0.30$ & $7.48 \pm 0.96 *$ & $6.55 \pm 0.30 *$ & $7.18 \pm 0.52 *$ & $7.33 \pm 1.37^{*}$ & $6.83 \pm 0.80 *$ \\
\hline \multirow[t]{2}{*}{ HEC } & 0 & $2.78 \pm 0.06$ & $2.75 \pm 0.30$ & $2.75 \pm 0.15$ & $3.18 \pm 0.45$ & $3.35 \pm 0.13$ & $3.10 \pm 0.28$ \\
\hline & 2 & $3.03 \pm 0.22$ & $6.13 \pm 0.66^{*}$ & $6.35 \pm 0.25^{*}$ & $5.33 \pm 0.23 *$ & $5.58 \pm 0.13^{*}$ & $6.48 \pm 0.18^{*}$ \\
\hline
\end{tabular}

Each value represents mean \pm SEM $(n=4) .{ }^{*} p<0.05$ compared with each control group. $P E G$ polyethylene glycol, $H E C$ hydroxyethylcellulose. 
experiment, the poloxamer base was more mucoadhesive to the rectal mucous membrane than that of hydroxyethylcellulose base. This indicates that the preparation using poloxamer as the rectal gel base might result in a strong contact with the rectal mucosa membrane than that of hydroxyethylcellulose as rectal gel base.

In our in vitro release experiment, distilled water was used as the dissolution medium because of its low-buffer capacity [17]. Then, tramadol hydrochloride was completely released from polyethylene glycol suppository base within 15 minutes. This fast release might be due to the erosion mechanism of this base [18]. Similarly, tramadol hydrochloride was released rapidly from the Witepsol H15 suppository base. This might be due to a rapid melting of base and a low affinity of drug to the base [7]. Since the amount of tramadol hydrochloride release from the suppository base was about $93 \%$ at 120 minutes, the rest of this drug might be dissolved in the Witepsol H15 base. In addition, tramadol hydrochloride was released from both gel bases rapidly probably due to both the tramadol hydrochloride and the bases being soluble in water.

A hot plate test was used to evaluate the central analgesic action of the drug [19]. The dose of tramadol $(2 \mathrm{mg})$ used in each rat corresponded to an analgesic dose of $10 \mathrm{mg} / \mathrm{kg}$ tramadol [20]. Rectal administration of tramadol using Witepsol H15 or polyethylene glycol suppository base significantly prolonged the latency of the nociceptive response in rats. This might be due to the fast release of tramadol. Nevertheless, we must note that slightly more pronounced analgesic effect appeared with the polyethylene glycol base than with the Witepsol H15 based suppositories. For the rectal gel, similar results were observed when using poloxamer or hydroxyethylcellulose as gel base. However, there was slightly less pronounced analgesic effect with the hydroxyethylcellulose base than with the poloxamer base in the rats. These might be due to the higher gel strength and more mucoadhesive force to the rectal mucous membrane of the poloxamer base than that of the hydroxyethylcellulose base, as shown in the present in vitro experiment.

In conclusion, tramadol hydrochloride suppositories and rectal gels prepared using different bases showed a rapid and almost complete release of the drug from their bases, prolonging the latency of the nociceptive response in in vivo experiments. Rectal administration of tramadol in addition to oral and intravenous administration may be an alternative route for the treatment of pain.

\section{Acknowledgement}

This research is supported by a grant from Prince of Songkla University. The authors have no conflict of interest to report.

\section{References}

1. Dayer P, Collart L, Desmeules J. The pharmacology of tramadol. Drugs. 1994; 47(Suppl):3-7.

2. Grond S, Sablotzki A. Clinical pharmacology of tramadol. Clin Pharmacokinet. 2004; 43:879-923.

3. Micromedex Healthcare Series: Document 2007. Tramadol. Available from: http://www.thomsonhc.com/ hcs/librarian/ND_PR/Main/SBK/3/PFPUI/7z16hW4.

4. Petrone D, Kamin M, Olson W. Slowing the titration rate of tramadol $\mathrm{HCl}$ reduces the incidence of discontinuation due to nausea and/ or vomiting: a double-blind randomized trial. J Clin Pharm Ther. 1999; 24:115-23.

5. Mercadante S, Arcuri E, Fusco F, Tirelli W, Villari P, Bussolino C, Campa T, De Conno F, Ripamonti C. Randomized double-blind, double-dummy crossover clinical trial of oral tramadol versus rectal tramadol administration in opioid-na ve cancer patients with pain. Support Care Cancer. 2005; 13:702-7.

6. Allen LV Jr. Compounding suppositories. Part I: theoretical considerations. Int J Pharm Compound. 2000; 4:289-93.

7. MIMS Thailand Index of Medical Specialties, $115^{\text {th }}$ Edition, 2009.

8. British Pharmacopoeia 2007. CD version 11.0.

9. Yong CS, Oh YK, Jung SH, Rhee JD, Kim HD, Kim CK, Choi JS, Choi HG. Preparation of ibuprofen-loaded liquid suppository using eutectic mixture system with menthol. Eur J Pharm Sci. 2004; 23:347-53.

10. Woolfe G, MacDonald AD. The evaluation of the analgesic action of pethidine hydrochloride (Demerol). J Pharmacol Exp Ther. 1944; 80:300-7.

11. Yun MO, Choi HG, Jung JH, Kim CH. Development of a thermo-reversible insulin liquid suppository with bioavailability enhancement. Int J Pharm. 1999; 189: 137-45.

12. Allen, LV Jr. Featured excipient: The poloxamers. Int J Pharm Compound. 2002; 6:58-9.

13. Miller SC, Drabik BR Rheological properties of poloxamer vehicles. Int J Pharm. 1984; 18:269-79.

14. Choi HG, Lee MK, Kim MH, Kim CK. Effects of additives on the physicochemicasl properties of 
liquid suppository bases. Int J Pharm.1999; 190:13-9.

15. Barakat NS. In vitro and in vivo charecteristics of thermogelling rectal drug delivery. AAPS PharmSciTech. 2004; 10:724-31.

16. Choi HG, Oh YK, Kim CK. In-situ gelling and mucoadhesive liquid suppository containing acetaminophen: enhanced bioavailability. Int J Pharm. 1998; 165:23-32.

17. Hewitt WD. Compounded suppositories and pharmaceutical care. Int J Pharm Compound. 1997; 1: 93-9.
18. Hosny EA, Abdeel-Hady SS, El-Tahir K. Formulation, in vitro release and ex vivo spasmolytic effects of mebeverine hydrochloride suppositories containing polycarbophil or polysorbate 80. Int J Pharm. 1996; 142:163-8.

19. LeBars D, Gozariu M, Cadden SW. Animal models of nociception. Pharmacol Rev. 2001; 53:597-652.

20. Sacerdote P, Bianchi M, Manfredi B, Panerai AE. Effects of tramadol on immune response and nociceptive thresholds in mice. Pain. 1997; 72:325-30. 